\title{
Vertex Exponent of Asymmetric Two-coloured Cycle
}

\section{A. Syahmarani ${ }^{*}$ and S. Suwilo ${ }^{1}$}

${ }^{1}$ Department of Mathematics, Universitas Sumatera Utara, Medan, 20155, Indonesia

\begin{abstract}
This paper is about an asymmetric two-coloured cycle. Let $D$ be an asymmetric two-coloured cycle on $n$ vertices, where $n$ is odd and $n \geq 3$, we show that the exponent of the $k$ th vertex of $D$ is exactly $\left(n^{2}-1\right) / 4+\lfloor k / 2\rfloor$.
\end{abstract}

Keywords: Exponent Digraphs, Primitive, Two-coloured Digraphs, Vertex Exponent.

Abstrak. Paper ini membahas tentang cycle dwi-warna asimetrik. Misalkan D merupakan suatu cycle dwi-warna asimetrik dengan $n$ vertex, dimana $n$ ganjil dan $n \geq 3$, diperlihatkan bahwa eksponen vertex ke $k$ dari $D$ adalah tepat $\left(n^{2}-1\right) / 4+\lfloor k / 2\rfloor$.

Kata Kunci: Eksponen Digraph, Primitif, Digraph Dwi-warna, Eksponen Vertex.

Received 20 July 2020 | Revised 13 August 2020 | Accepted 8 September 2020

\section{Digraphs and Two-coloured Digraphs}

We discuss about vertex exponent of primitive asymmetric two-coloured digraphs with a special type. The notations and terminologies for digraphs and two-coloured digraphs in this paper is based on [1]. A walk of length $m$ from vertex $u$ to vertex $v$ in a graph is a sequence of $m$ of the form

$$
\left(v_{0}, v_{1}\right),\left(v_{1}, v_{2}\right), \ldots,\left(v_{m-1}, v_{m}\right)
$$

where $v_{0}=u$ and $v_{m}=v$. We state a walk $w$ from $u$ to $v$ as a $(u, v)$-walk or $w_{u v}$ and $\ell\left(w_{u v}\right)$ is denoted by its length. A $(u, v)$-walk is closed provided $u=v$ and if not is open. A path from $u$ to $v$ is a walk with no repeated vertices except possibly $u=v$. A cycle is a closed path. A loop is a closed cycle of length 1 .

A digraph $D$ called strongly connected if for each pair of vertices $u$ and $v$ there is a $(u, v)$-walk and also a $(v, u)$-walk of length exactly $k$ in $D$. The exponent of $D$, denoted by $\exp (D)$, is the smallest of such positive integer $k$. A strongly connected digraph $D$ is primitive if and only if the greatest common divisor of lengths of all cycle in $D$ is 1 [1]. A symmetric digraph $D$ is a digraph such that the $\operatorname{arc}(u, v)$ is in $D$ whenever the $\operatorname{arc}(v, u)$ is in $D$. Since a symmetric digraph must have a cycle of length 2 , a symmetric digraph is primitive if and only if it contains a cycle with odd length.

*Corresponding author at: Department of Mathematics, Universitas Sumatera Utara, Medan, 20155, Indonesia

E-mail address: aghni.syahmarani@usu.ac.id 
A two-coloured digraph or a 2-digraph, is a digraph in which each of its arcs is coloured by either red or blue ( 2 colours). In a two-coloured digraph we differentiate a walk by how many red and how many blue arcs it contains. We intend an $(h, k)^{T}$-walk from $u$ to $v$ as a $(u, v)$-walk that consists of $h$ red arcs and $k$ blue arcs and the length is $h+k$. The vector $(r(w), b(w))^{T}$ is called the composition of the walk $w$. A two-coloured digraph $D$ is strongly connected provided that its underlying digraph is strongly connected. Underlying digraph means the digraph obtained from $D$ by ignoring its arc colour. An asymmetric two-coloured digraph is a symmetric two-coloured digraph for which an arc $(u, v)$ is coloured by red whenever the $\operatorname{arc}(v, u)$ is coloured by blue and vice versa. A strongly connected two-coloured digraph is primitive provided there are nonnegative integers $h$ and $k$ such that for each pair of vertices $u$ and $v$ there is an $(h, k)^{T}$-walk from $u$ to $v$. The smallest nonnegative integer $h+k$ among all such nonnegative integers $h$ and $k$ is called the 2-exponent of $D$, denoted by $\exp _{2}(D)$.

Let a two-coloured digraph $D$ and $\left\{\delta=\delta_{1}, \delta_{2}, \ldots, \delta_{t}\right\}$ as its set of all cycle. A cycle matrix $M$ of $D$ is a 2 by $t$ matrix whose $i$ th column is the composition of the cycle $\delta_{i}, i=1,2, \ldots, t$. That is

$$
M=\left[\begin{array}{llll}
r\left(\delta_{1}\right) & r\left(\delta_{2}\right) & \ldots & r\left(\delta_{t}\right) \\
b\left(\delta_{1}\right) & b\left(\delta_{2}\right) & \ldots & b\left(\delta_{t}\right)
\end{array}\right]
$$

The content of $M$ is defined to be 0 if the $\operatorname{rank}(M)=1$ and the greatest common divisor of the 2 by 2 minors of $M$, otherwise.

Shader and Suwilo [1] initiated the research on 2-exponents of two-coloured digraphs. They indicated that the largest 2-exponent of primitive two-coloured digraphs on $n$ vertices lies on the interval $\left[\left(n^{3}-5 n^{2}\right) / 3,\left(3 n^{3}+2 n^{2}-2 n\right) / 2\right]$. Since that time, many papers have been published on the subject. Suwilo [2] has shown the exponent of an asymmetric primitive two-coloured $(n, s)$ lollipop. Let $D$ be an asymmetric primitive two-coloured $(n, s)$-lollipop. Since $D$ has a red path of length $(s+1) / 2+(n-s)$, then $\exp _{2}(D)=\left(s^{2}-1\right) / 2+(s+1)(n-s)$. Suwilo has also shown that if $n$ is odd and $s=n$ or $s=n-2$, then $\exp _{2}(D)=\left(n^{2}-1\right) / 2$ and if $n$ is even and $s=n-1$, then $\exp _{2}(D)=n^{2} / 2$. Gao and Shao [3] have shown the generalized exponent of primitive twocoloured Wielandt digraph $W_{n}^{(2)}$. They showed 3 types of Wielandt digraph and found the formula to find vertex exponent for each type. Fomichev and Avezova [4] found the exact formula for the exponents of the mixing digraphs of register transformations.

Suwilo [5] also found the vertex exponent of two-colored primitive extremal ministrong digraphs $D^{(2)}$ on $n$ vertices. If $D^{(2)}$ has one blue arc, he showed that the exponents of vertices of $D^{(2)}$ lie on $\left[n^{2}-5 n+8, n^{2}-3 n+1\right]$. Since $D^{(2)}$ has two blue arcs, he also showed that the exponents of vertices in $D^{(2)}$ lie on $\left[n^{2}-4 n+4, n^{2}-n\right]$. Vertex exponents of a class of two-colored of the Hamiltonian digraphs have been shown in [6]. They found that the vertex exponents of primitive two-colored digraph $L_{n}^{(2)}$ on $n \geq 5$ vertices whose underlying digraph is the Hamiltonian digraph consisting of the cycle $v_{1} \rightarrow v_{n} \rightarrow v_{n-1} \rightarrow \cdots \rightarrow v_{2} \rightarrow v_{1}$ and the arc $v_{1} \rightarrow v_{n-2}$ is known that $2 n^{2}-6 n+2 \geq \exp \left(L_{n}^{(2)}\right) \geq\left(n^{3}-2 n^{2}+1\right) / 2$. If the $\exp \left(L_{n}^{(2)}\right)=\left(n^{3}-2 n^{2}+1\right) / 2$, then its vertex exponents lie on $\left[\left(n^{3}-2 n^{2}-3 n+4\right) / 4,\left(n^{3}-2 n^{2}+3 n+6\right) / 4\right]$ and if $\exp \left(L_{n}^{(2)}\right)=2 n^{2}-6 n+2$, then its vertex exponents lie on $\left[n^{2}-4 n+5, n^{2}-2 n-1\right]$.

The aim of this paper is to show the vertex exponent of an asymmetric primitive two-coloured cycle. We give the formula to find the vertex exponent of an asymmetric primitive two-coloured 
cycle as the result.

\section{Vertex Exponent of An Asymmetric Two-coloured Cycle}

Let $D$ be an asymmetric primitive two-coloured cylce of length $n$. If $D$ is primitive, then $n$ have to be odd and $n \geq 3$. Furthermore, if $D$ is asymmetric, then $D$ has directed cycles of length 2 with composition $(1,1)^{T}$, and also has two directed cycles $\delta_{1}$ and $\delta_{2}$ of length $n$. The cycle are

$$
(1,2),(2,4), \ldots,(n-1, n),(n, n-2), \ldots,(5,3),(3,1)
$$

and

$$
(1,3),(3,5), \ldots,(n, n-1),(n-1, n-3), \ldots,(4,2),(2,1) .
$$

Hence, the compositions form of the directed cycles of $D$ are $(1,1)^{T},(n-a, a)^{T}$, and $(a, n-a)^{T}$ for some nonnegative integer $0 \leq a \leq n$. This implies the cycle matrix of $D$ is of the form

$$
M=\left[\begin{array}{cccccc}
n-a & a & 1 & 1 & \ldots & 1 \\
a & n-a & 1 & 1 & \ldots & 1
\end{array}\right]
$$

Since $D$ is primitive, by [1] the content of $M$ is 1 . Therefore

$$
1=\operatorname{gcd}(n(n-2 a), n-2 a, 2 a-n)= \pm(n-2 a) .
$$

This implies that $a=(n+1) / 2$ or $a=(n-1) / 2$, so without loss the generality we may assume that

$$
M=\left[\begin{array}{lllllll}
(n+1) / 2 & (n-1) / 2 & 1 & 1 & \ldots & 1 \\
(n-1) / 2 & (n+1) / 2 & 1 & 1 & \ldots & 1
\end{array}\right] .
$$

We note that since $D$ is asymmetric, every vertex of $D$ lies on a $(1,1)^{T}$-walk. This means each $(r, b)^{T}$-walk in $D$ can be extended to a $(r+t, b+t)^{T}$-walk for each positive integer $t \geq 1$.

Let $D$ be an asymmetric primitive two-coloured cycle where the arcs colouring in succession are $(n+1) / 2$ red arcs and $(n-1) / 2$ blue arcs or vice versa. Since $n$ is odd, there are two cases. For $n=4 m+1$, colour $\gamma_{1}$ by giving red on $\left(v_{j}, v_{j-2}\right)$-arcs where $3 \leq j \leq(n+1) / 2,\left(v_{1}, v_{2}\right)$-arc, and $\left(v_{i}, v_{i+2}\right)$-arcs where $2 \leq i \leq(n-1) / 2$ and the others with blue. For $n=4 m+3$, colour $\gamma_{1}$ by giving red on $\left(v_{j}, v_{j-2}\right)$-arcs where $3 \leq j \leq(n+3) / 2,\left(v_{1}, v_{2}\right)$-arc, and $\left(v_{i}, v_{i+2}\right)$-arcs where $2 \leq i \leq(n+1) / 2$ and the others with blue.

Lemma 2.1. Suppose $D$ be an asymmetric primitive two-coloured cycle of length $n$, then the exponent $v_{k}$ of $D \exp _{D}\left(v_{k}\right) \geq \frac{1}{4}\left(n^{2}-1\right)+\left\lfloor\frac{k}{2}\right\rfloor$.

Proof. Suppose $D$ be an asymmetric primitive two-coloured cylce of length $n$. Since $D$ is primitive, then $n$ have to be odd and $n \geq 3$. Moreover, since $D$ is asymmetric, for each $v_{k}$ on $D$ there is a $(1,1)^{T}$-closed walk from $v_{k}$ to itself of length 2 . Let $p_{k, x}$ be a path which connect $v_{k}$ to $v_{x}$ on $D$ is a $\left(r_{p_{k, x}}, b_{p_{k, x}}\right)^{T}$-path consist $r_{p_{k, x}}$ red arcs and $b_{p_{k, x}}$ blue arcs. For each $v_{k}$ on $D$, then $(r, b)^{T}$-walk from $v_{k}$ to $v_{x}$ is of the form

$$
\left[\begin{array}{l}
r \\
b
\end{array}\right]=\left[\begin{array}{c}
r_{p_{k, x}} \\
b_{p_{k, x}}
\end{array}\right]+x_{1}\left[\begin{array}{c}
\frac{n+1}{2} \\
\frac{n-1}{2}
\end{array}\right]+x_{2}\left[\begin{array}{c}
\frac{n-1}{2} \\
\frac{n+1}{2}
\end{array}\right]+x_{3}\left[\begin{array}{c}
1 \\
1
\end{array}\right]
$$


for some nonnegative integers $x_{1}, x_{2}$ and $x_{3}$.

Case 1. For $n=4 m+1$, we show that $\exp _{D}\left(v_{k}\right) \geq \frac{1}{4}\left(n^{2}-1\right)+\left\lfloor\frac{k}{2}\right\rfloor$. If $k$ is even, for each $v_{x}$ on $D$, there is a $(r, b)^{T}$-walk from $v_{k}$ to $v_{x}$. We take a $(r, b)^{T}$-walk from $v_{k}$ to $v_{\frac{n-1}{2}}$ and to $v_{\frac{n+1}{2}}$, then we find $\exp _{D}\left(v_{k}\right) \geq \frac{1}{4}\left(n^{2}-1\right)+\frac{k}{2}$.

There are red path of length $e$ and blue path of length $f$ from $v_{k}$ to $v_{\frac{n-1}{2}}$, then the $(r, b)^{T}$-walk is of the form

$$
\left[\begin{array}{l}
r \\
b
\end{array}\right]=\left[\begin{array}{l}
e \\
f
\end{array}\right]+x_{1}\left[\begin{array}{c}
\frac{n+1}{2} \\
\frac{n-1}{2}
\end{array}\right]+x_{2}\left[\begin{array}{c}
\frac{n-1}{2} \\
\frac{n+1}{2}
\end{array}\right]+x_{3}\left[\begin{array}{l}
1 \\
1
\end{array}\right]
$$

for some nonnegative integers $e, f, x_{1}, x_{2}$ and $x_{3}$.

There are also red path of length $g$ and blue path of length $h$ from $v_{k}$ to $v_{\frac{n+1}{2}}$, then the $(r, b)^{T}$-walk is of the form

$$
\left[\begin{array}{l}
r \\
b
\end{array}\right]=\left[\begin{array}{l}
g \\
h
\end{array}\right]+y_{1}\left[\begin{array}{c}
\frac{n+1}{2} \\
\frac{n-1}{2}
\end{array}\right]+y_{2}\left[\begin{array}{c}
\frac{n-1}{2} \\
\frac{n+1}{2}
\end{array}\right]+y_{3}\left[\begin{array}{l}
1 \\
1
\end{array}\right]
$$

for some nonnegative integers $g, h, y_{1}, y_{2}$ and $y_{3}$. We note that if $1 \leq k<\frac{n+1}{2}$, then $e=\frac{n-1}{4}-\frac{k}{2}$, $f=0, g=0$ and $h=\frac{n-1}{4}+\frac{k}{2}$. But if $\frac{n+1}{2} \leq k \leq n$, then $e=g=\frac{k}{2}-\frac{n-1}{4}, f=0$, and $h=\frac{n-1}{2}$. By setting both of those equations we have

$$
\left[\begin{array}{l}
e-g \\
f-h
\end{array}\right]=\left(y_{1}-x_{1}\right)\left[\begin{array}{c}
\frac{n+1}{2} \\
\frac{n-1}{2}
\end{array}\right]+\left(y_{2}-x_{2}\right)\left[\begin{array}{c}
\frac{n-1}{2} \\
\frac{n+1}{2}
\end{array}\right]+\left(y_{3}-x_{3}\right)\left[\begin{array}{l}
1 \\
1
\end{array}\right]
$$

Substraction the second by the first component of Equation 2.1, then we have $\left(y_{1}-x_{1}\right)+\left(x_{2}-\right.$ $\left.y_{2}\right)=2\left(\frac{n-1}{4}\right)$. This implies $y_{1}+x_{2} \geq 2\left(\frac{n-1}{4}\right)$ and hence $y_{1} \geq \frac{n-1}{4}$ or $x_{2} \geq \frac{n-1}{4}$. Hence we now have that $\exp _{D}\left(v_{k}\right) \geq \frac{1}{4}\left(n^{2}-1\right)+\frac{k}{2}$.

If $k$ is odd, for each $v_{x}$ on $D$, there is a $(r, b)^{T}$-walk from $v_{k}$ to $v_{x}$. We take a $(r, b)^{T}$-walk from $v_{k}$ to $v_{\frac{n-1}{2}}$ and to $v_{\frac{n+1}{2}}$, then we find $\exp _{D}\left(v_{k}\right) \geq \frac{1}{4}\left(n^{2}-1\right)+\frac{k-1}{2}$.

There are red path of length $e$ and blue path of length $f$ from $v_{k}$ to $v_{\frac{n-1}{2}}$, then the $(r, b)^{T}$-walk is of the form

$$
\left[\begin{array}{l}
r \\
b
\end{array}\right]=\left[\begin{array}{l}
e \\
f
\end{array}\right]+x_{1}\left[\begin{array}{c}
\frac{n+1}{2} \\
\frac{n-1}{2}
\end{array}\right]+x_{2}\left[\begin{array}{c}
\frac{n-1}{2} \\
\frac{n+1}{2}
\end{array}\right]+x_{3}\left[\begin{array}{l}
1 \\
1
\end{array}\right]
$$

for some nonnegative integers $e, f, x_{1}, x_{2}$ and $x_{3}$.

There are also red path of length $g$ and blue path of length $h$ from $v_{k}$ to $v_{\frac{n+1}{2}}$, then the $(r, b)^{T}$-walk is of the form

$$
\left[\begin{array}{l}
r \\
b
\end{array}\right]=\left[\begin{array}{l}
g \\
h
\end{array}\right]+y_{1}\left[\begin{array}{c}
\frac{n+1}{2} \\
\frac{n-1}{2}
\end{array}\right]+y_{2}\left[\begin{array}{c}
\frac{n-1}{2} \\
\frac{n+1}{2}
\end{array}\right]+y_{3}\left[\begin{array}{l}
1 \\
1
\end{array}\right]
$$

for some nonnegative integers $g, h, y_{1}, y_{2}$ and $y_{3}$. We note that if $1 \leq k \leq \frac{n+1}{2}$, then $e=\frac{n-1}{4}+\frac{k-1}{2}$, $f=0, g=0$ and $h=\frac{n-1}{4}-\frac{k-1}{2}$. But if $\frac{n+1}{2}<k \leq n$, then $e=\frac{n-1}{2} f=\frac{k-1}{2}-\frac{n-1}{4}, g=0$, and $h=\frac{k-1}{2}-\frac{n-1}{4}$. By setting both of those equations we have

$$
\left[\begin{array}{c}
e-g \\
f-h
\end{array}\right]=\left(y_{1}-x_{1}\right)\left[\begin{array}{c}
\frac{n+1}{2} \\
\frac{n-1}{2}
\end{array}\right]+\left(y_{2}-x_{2}\right)\left[\begin{array}{c}
\frac{n-1}{2} \\
\frac{n+1}{2}
\end{array}\right]+\left(y_{3}-x_{3}\right)\left[\begin{array}{l}
1 \\
1
\end{array}\right]
$$


Substraction the second by the first component of Equation 2.2, then we have $\left(y_{1}-x_{1}\right)+\left(x_{2}-\right.$ $\left.y_{2}\right)=2\left(\frac{n-1}{4}\right)$. This implies $y_{1}+x_{2} \geq 2\left(\frac{n-1}{4}\right)$ and hence $y_{1} \geq \frac{n-1}{4}$ or $x_{2} \geq \frac{n-1}{4}$. Hence we now have that $\exp _{D}\left(v_{k}\right) \geq \frac{1}{4}\left(n^{2}-1\right)+\frac{k-1}{2}$. Then for each $k$ where $1 \leq k \leq n$ we have $\exp _{D}\left(v_{k}\right) \geq$ $\frac{1}{4}\left(n^{2}-1\right)+\left\lfloor\frac{k}{2}\right\rfloor$.

Case 2. For $n=4 m+3$, we also show that $\exp _{D}\left(v_{k}\right) \geq \frac{1}{4}\left(n^{2}-1\right)+\left\lfloor\frac{k}{2}\right\rfloor$. If $k$ is even, for each $v_{x}$ on $D$, there is a $(r, b)^{T}$-walk from $v_{k}$ to $v_{x}$. We take a $(r, b)^{T}$-walk from $v_{k}$ to $v_{\frac{n+1}{2}}$ and to $v_{\frac{n+3}{2}}$, then we find $\exp _{D}\left(v_{k}\right) \geq \frac{1}{4}\left(n^{2}-1\right)+\frac{k}{2}$.

There are red path of length $e$ and blue path of length $f$ from $v_{k}$ to $v_{\frac{n+1}{2}}$, then the $(r, b)^{T}$-walk is of the form

$$
\left[\begin{array}{l}
r \\
b
\end{array}\right]=\left[\begin{array}{l}
e \\
f
\end{array}\right]+x_{1}\left[\begin{array}{c}
\frac{n+1}{2} \\
\frac{n-1}{2}
\end{array}\right]+x_{2}\left[\begin{array}{c}
\frac{n-1}{2} \\
\frac{n+1}{2}
\end{array}\right]+x_{3}\left[\begin{array}{l}
1 \\
1
\end{array}\right]
$$

for some nonnegative integers $e, f, x_{1}, x_{2}$ and $x_{3}$.

There are also red path of length $g$ and blue path of length $h$ from $v_{k}$ to $v_{\frac{n+3}{2}}$, then the $(r, b)^{T}$-walk is of the form

$$
\left[\begin{array}{l}
r \\
b
\end{array}\right]=\left[\begin{array}{l}
g \\
h
\end{array}\right]+y_{1}\left[\begin{array}{c}
\frac{n+1}{2} \\
\frac{n-1}{2}
\end{array}\right]+y_{2}\left[\begin{array}{c}
\frac{n-1}{2} \\
\frac{n+1}{2}
\end{array}\right]+y_{3}\left[\begin{array}{c}
1 \\
1
\end{array}\right]
$$

for some nonnegative integers $g, h, y_{1}, y_{2}$ and $y_{3}$. We note that if $1 \leq k \leq \frac{n+1}{2}$, then $e=\frac{n-1}{2}$, $f=\frac{n+1}{4}+\frac{k}{2}, g=\frac{n+1}{4}-\frac{k}{2}$ and $h=\frac{n-1}{2}$. But if $\frac{n+1}{2}<k \leq n$, then $e=\frac{k}{2}-\frac{n-1}{4}, f=0, g=0$ and $h=\frac{3 n-1}{4}-\frac{k}{2}$. By setting both of those equations we have

$$
\left[\begin{array}{c}
e-g \\
f-h
\end{array}\right]=\left(y_{1}-x_{1}\right)\left[\begin{array}{c}
\frac{n+1}{2} \\
\frac{n-1}{2}
\end{array}\right]+\left(y_{2}-x_{2}\right)\left[\begin{array}{c}
\frac{n-1}{2} \\
\frac{n+1}{2}
\end{array}\right]+\left(y_{3}-x_{3}\right)\left[\begin{array}{l}
1 \\
1
\end{array}\right]
$$

Substraction the second by the first component of Equation 2.3, then we have $\left(y_{1}-x_{1}\right)+\left(x_{2}-\right.$ $\left.y_{2}\right)=2\left(\frac{n-3}{4}\right)$. This implies $y_{1}+x_{2} \geq 2\left(\frac{n-3}{4}\right)$ and hence $y_{1} \geq \frac{n-3}{4}$ or $x_{2} \geq \frac{n-3}{4}$. Hence we now have that $\exp _{D}\left(v_{k}\right) \geq \frac{1}{4}\left(n^{2}-1\right)+\frac{k}{2}$.

If $k$ is odd, for each $v_{x}$ on $D$, there is a $(r, b)^{T}$-walk from $v_{k}$ to $v_{x}$. We take a $(r, b)^{T}$-walk from $v_{k}$ to $v_{\frac{n+1}{2}}$ and to $v_{\frac{n+3}{2}}$, then we find $\exp _{D}\left(v_{k}\right) \geq \frac{1}{4}\left(n^{2}-1\right)+\frac{k-1}{2}$.

There are red path of length $e$ and blue path of length $f$ from $v_{k}$ to $v_{\frac{n+1}{2}}$, then the $(r, b)^{T}$-walk is of the form

$$
\left[\begin{array}{l}
r \\
b
\end{array}\right]=\left[\begin{array}{l}
e \\
f
\end{array}\right]+x_{1}\left[\begin{array}{c}
\frac{n+1}{2} \\
\frac{n-1}{2}
\end{array}\right]+x_{2}\left[\begin{array}{c}
\frac{n-1}{2} \\
\frac{n+1}{2}
\end{array}\right]+x_{3}\left[\begin{array}{l}
1 \\
1
\end{array}\right]
$$

for some nonnegative integers $e, f, x_{1}, x_{2}$ and $x_{3}$.

There are also red path of length $g$ and blue path of length $h$ from $v_{k}$ to $v_{\frac{n+3}{2}}$, then the $(r, b)^{T}$-walk is of the form

$$
\left[\begin{array}{l}
r \\
b
\end{array}\right]=\left[\begin{array}{l}
g \\
h
\end{array}\right]+y_{1}\left[\begin{array}{c}
\frac{n+1}{2} \\
\frac{n-1}{2}
\end{array}\right]+y_{2}\left[\begin{array}{c}
\frac{n-1}{2} \\
\frac{n+1}{2}
\end{array}\right]+y_{3}\left[\begin{array}{c}
1 \\
1
\end{array}\right]
$$

for some nonnegative integers $g, h, y_{1}, y_{2}$ and $y_{3}$. We note that if $1 \leq k \leq \frac{n+1}{2}$, then $e=\frac{3 n-1}{4}+\frac{k-1}{2}$, $f=0, g=0$ and $h=\frac{k-1}{2}-\frac{n+1}{4}$. But if $\frac{n+1}{2}<k \leq n$, then $e=\frac{k-1}{2}-\frac{n+1}{4}, f=0, g=0$, and 
$h=\frac{k-1}{2}-\frac{3 n-1}{4}$. By setting both of those equations we have

$$
\left[\begin{array}{c}
e-g \\
f-h
\end{array}\right]=\left(y_{1}-x_{1}\right)\left[\begin{array}{c}
\frac{n+1}{2} \\
\frac{n-1}{2}
\end{array}\right]+\left(y_{2}-x_{2}\right)\left[\begin{array}{c}
\frac{n-1}{2} \\
\frac{n+1}{2}
\end{array}\right]+\left(y_{3}-x_{3}\right)\left[\begin{array}{l}
1 \\
1
\end{array}\right]
$$

Substraction the second by the first component of Equation 2.4, then if $1 \leq k \leq \frac{n+1}{2}$, we have $\left(y_{1}-x_{1}\right)+\left(x_{2}-y_{2}\right)=2\left(\frac{n-3}{4}\right)$. This implies $y_{1}+x_{2} \geq 2\left(\frac{n-3}{4}\right)$ and hence $y_{1} \geq \frac{n-3}{4}$ or $x_{2} \geq \frac{n-3}{4}$. And if $\frac{n+1}{2}<k \leq n$, we have $\left(y_{1}-x_{1}\right)+\left(x_{2}-y_{2}\right)=2\left(\frac{n-1}{4}\right)$. This implies $y_{1}+x_{2} \geq 2\left(\frac{n-1}{4}\right)$ and hence $y_{1} \geq \frac{n-1}{4}$ or $x_{2} \geq \frac{n-1}{4}$. Hence we now have that $\exp _{D}\left(v_{k}\right) \geq \frac{1}{4}\left(n^{2}-1\right)+\frac{k-1}{2}$. Then for each $k$ where $1 \leq k \leq n$ we have $\exp _{D}\left(v_{k}\right) \geq \frac{1}{4}\left(n^{2}-1\right)+\left\lfloor\frac{k}{2}\right\rfloor$.

Lemma 2.2. Let $D$ be an asymmetric primitive two-coloured cycle of length $n$, then the exponent $v_{k}$ of $D \exp _{D}\left(v_{k}\right) \leq \frac{1}{4}\left(n^{2}-1\right)+\left\lfloor\frac{k}{2}\right\rfloor$.

Proof. Let $D$ be an asymmetric primitive two-coloured cylce of length $n$. If $D$ is primitive, then $n$ have to be odd and $n \geq 3$. Moreover, if $D$ is asymmetric, for each $v_{k}$ on $D$ there is a $(1,1)^{T}$-closed walk from $v_{k}$ to itself of length 2 . For $k=1$, there is a $(r, b)^{T}$-walk from $v_{1}$ to $v_{j}$ with composition $\left(\frac{n^{2}-1}{8}, \frac{n^{2}-1}{8}\right)^{T}$. We show that $\exp _{D}\left(v_{1}\right)=\frac{1}{4}\left(n^{2}-1\right)$. First, we show $\exp _{D}\left(v_{1}\right) \leq \frac{1}{4}\left(n^{2}-1\right)$. Let $P_{1, j}$ be a path which start from $v_{1}$ to $v_{j}$. There is a $(r, b)^{T}$-walk from $v_{1}$ to $v_{j}$. In this case, the walk that starts at $v_{1}$, moves to $v_{j}$ along the path $P_{1, j}$, and moves $\left(r\left(p_{1, j}\right)-b\left(p_{1, j}\right)\right)$ times around the cycle $\gamma_{1}$ is the shortest walk from $v_{1}$ to $v_{j}$. This composition of this walk is

$$
\left[\begin{array}{l}
r \\
b
\end{array}\right]=\left[\begin{array}{c}
\left(r\left(p_{1, j}\right)\right. \\
b\left(p_{1, j}\right)
\end{array}\right]+\left(r\left(p_{1, j}\right)-b\left(p_{1, j}\right)\right)\left[\begin{array}{c}
\frac{n-1}{2} \\
\frac{n+1}{2}
\end{array}\right] .
$$

Notice that $P_{1, j}$ is a path from $v_{1}$ to $v_{j}$ where $b\left(p_{1, j}\right)=0$. Then the composition of its walk becomes

$$
\left[\begin{array}{l}
r \\
b
\end{array}\right]=\left(r\left(p_{1, j}\right)-b\left(p_{1, j}\right)\right)\left[\begin{array}{c}
\frac{n+1}{2} \\
\frac{n+1}{2}
\end{array}\right] .
$$

We find that $P_{1, j} \leq \frac{n-1}{4}$. This implies $r\left(p_{1, j}\right)+b\left(p_{1, j}\right) \leq \frac{n-1}{4}$, hence $r\left(p_{1, j}\right)-b\left(p_{1, j}\right) \leq r\left(p_{1, j}\right)+$ $b\left(p_{1, j}\right) \leq \frac{n-1}{4}$, then

$$
\left[\begin{array}{l}
r \\
b
\end{array}\right] \leq\left[\begin{array}{c}
\frac{n^{2}-1}{8} \\
\frac{n^{2}-1}{8}
\end{array}\right]
$$

Now using $(1,1)^{T}$-walks, we can extend the walk into $(t, t)^{T}$-walk with $t=\frac{n^{2}-1}{8}$ then

$$
\exp _{D}\left(v_{1}\right) \leq \frac{1}{4}\left(n^{2}-1\right)
$$

Next we show $\exp _{D}\left(v_{1}\right) \geq \frac{1}{4}\left(n^{2}-1\right)$. Let $D$ be an asymmetric primitive two-coloured cylce of length $n$. Since $D$ is primitive, then $n$ must be odd and $n \geq 3$. Furthermore, since $D$ is asymmetric, for $v_{1}$ on $D$ there is a $(1,1)^{T}$-closed walk from $v_{1}$ to itself of length 2 . Let $p_{1, j}$ be a path which connect $v_{1}$ to $v_{j}$ on $D$ is a $\left(r_{p_{1, j}}, b_{p_{1, j}}\right)^{T}$-path consist $r_{p_{1, j}}$ red arcs and $b_{p_{1, j}}$ blue arcs. For $v_{1}$ on $D$, then $(r, b)^{T}$-walk from $v_{1}$ to $v_{j}$ is of the form

$$
\left[\begin{array}{l}
r \\
b
\end{array}\right]=\left[\begin{array}{l}
r_{p_{1, j}} \\
b_{p_{1, j}}
\end{array}\right]+x_{1}\left[\begin{array}{c}
\frac{n+1}{2} \\
\frac{n-1}{2}
\end{array}\right]+x_{2}\left[\begin{array}{c}
\frac{n-1}{2} \\
\frac{n+1}{2}
\end{array}\right]+x_{3}\left[\begin{array}{l}
1 \\
1
\end{array}\right]
$$


for some nonnegative integers $x_{1}, x_{2}$ and $x_{3}$.

For each $v_{j}$ on $D$, there is a $(r, b)^{T}$-walk from $v_{1}$ to $v_{j}$. If $n=4 m+1$, we take a $(r, b)^{T}$-walk from $v_{1}$ to $v_{\frac{n-1}{2}}$ and to $v_{\frac{n+1}{2}}$. And if $n=4 m+3$, we take a $(r, b)^{T}$-walk from $v_{1}$ to $v_{\frac{n+1}{2}}$ and to $v_{\frac{n+3}{2}}$. Then we find $\exp _{D}\left(v_{k}\right) \geq \frac{1}{4}\left(n^{2}-1\right)$.

There are red path of length $e$ and blue path of length $f$ from $v_{k}$ to $v_{\frac{n-1}{2}}$ if $n=4 m+1$ and to $v_{\frac{n+1}{2}}$ if $n=4 m+3$, then the $(r, b)^{T}$-walk is of the form

$$
\left[\begin{array}{l}
r \\
b
\end{array}\right]=\left[\begin{array}{l}
e \\
f
\end{array}\right]+x_{1}\left[\begin{array}{c}
\frac{n+1}{2} \\
\frac{n-1}{2}
\end{array}\right]+x_{2}\left[\begin{array}{c}
\frac{n-1}{2} \\
\frac{n+1}{2}
\end{array}\right]+x_{3}\left[\begin{array}{l}
1 \\
1
\end{array}\right]
$$

for some nonnegative integers $e, f, x_{1}, x_{2}$ and $x_{3}$.

There are also red path of length $g$ and blue path of length $h$ from $v_{k}$ to $v_{\frac{n+1}{2}}$ if $n=4 m+1$ and to $v_{\frac{n+3}{2}}$ if $n=4 m+3$, then the $(r, b)^{T}$-walk is of the form

$$
\left[\begin{array}{l}
r \\
b
\end{array}\right]=\left[\begin{array}{l}
g \\
h
\end{array}\right]+y_{1}\left[\begin{array}{c}
\frac{n+1}{2} \\
\frac{n-1}{2}
\end{array}\right]+y_{2}\left[\begin{array}{c}
\frac{n-1}{2} \\
\frac{n+1}{2}
\end{array}\right]+y_{3}\left[\begin{array}{l}
1 \\
1
\end{array}\right]
$$

for some nonnegative integers $g, h, y_{1}, y_{2}$ and $y_{3}$. We note that if $n=4 m+1$, then $e=\frac{n-1}{4}, f=0$, $g=0$ and $h=\frac{n-1}{4}$. But if $n=4 m+3$, then $e=\frac{n-1}{2}, f=\frac{n+1}{4}, g=\frac{n+1}{4}$, and $h=\frac{n-1}{2}$. By setting both of those equations we have

$$
\left[\begin{array}{c}
e-g \\
f-h
\end{array}\right]=\left(y_{1}-x_{1}\right)\left[\begin{array}{c}
\frac{n+1}{2} \\
\frac{n-1}{2}
\end{array}\right]+\left(y_{2}-x_{2}\right)\left[\begin{array}{c}
\frac{n-1}{2} \\
\frac{n+1}{2}
\end{array}\right]+\left(y_{3}-x_{3}\right)\left[\begin{array}{l}
1 \\
1
\end{array}\right]
$$

Substraction the second by the first component of Equation 2.5, then we have if $n=4 m+1$, we have $\left(y_{1}-x_{1}\right)+\left(x_{2}-y_{2}\right)=2\left(\frac{n-1}{4}\right)$. This implies $y_{1}+x_{2} \geq 2\left(\frac{n-1}{4}\right)$ and hence $y_{1} \geq \frac{n-1}{4}$ or $x_{2} \geq \frac{n-1}{4}$. And if $n=4 m+3$, we have $\left(y_{1}-x_{1}\right)+\left(x_{2}-y_{2}\right)=2\left(\frac{n-3}{4}\right)$. This implies $y_{1}+x_{2} \geq 2\left(\frac{n-3}{4}\right)$ and hence $y_{1} \geq \frac{n-3}{4}$ or $x_{2} \geq \frac{n-3}{4}$. Hence we now have that $\exp _{D}\left(v_{k}\right) \geq \frac{1}{4}\left(n^{2}-1\right)$. Since we have $\exp _{D}\left(v_{k}\right) \leq \frac{1}{4}\left(n^{2}-1\right)$ and also $\exp _{D}\left(v_{k}\right) \geq \frac{1}{4}\left(n^{2}-1\right)$ imply that $\exp _{D}\left(v_{k}\right)=\frac{1}{4}\left(n^{2}-1\right)$.

For $k \neq 1$, there is a $(r, b)^{T}$-walk from $v_{k}$ to $v_{j}$ that starts from $v_{k}$ to $v_{1}$ and then continue from $v_{1}$ to $v_{j}$. Let $p_{k, 1}$ be a path from $v_{k}$ to $v_{1}$ of length $\left\lfloor\frac{k}{2}\right\rfloor$. We have the composition of $(r, b)^{T}$-walk from $v_{1}$ to $v_{j}$ is of the form

$$
\left[\begin{array}{l}
r \\
b
\end{array}\right]=\left[\begin{array}{c}
\frac{n^{2}-1}{8} \\
\frac{n^{2}-1}{8}
\end{array}\right]
$$

Then we find the $(r, b)^{T}$-walk from $v_{k}$ to $v_{j}$ is of the form

$$
\left[\begin{array}{l}
r \\
b
\end{array}\right] \leq\left\lfloor\frac{k}{2}\right\rfloor+\left[\begin{array}{c}
\frac{n^{2}-1}{8} \\
\frac{n^{2}-1}{8}
\end{array}\right]
$$

Then we have $\exp _{D}\left(v_{k}\right) \leq \frac{1}{4}\left(n^{2}-1\right)+\left\lfloor\frac{k}{2}\right\rfloor$.

Theorem 2.3. Let $D$ be an asymmetric primitive two-coloured cycle of length $n$, then the exponent $v_{k}$ of $D \exp _{D}\left(v_{k}\right)=\frac{1}{4}\left(n^{2}-1\right)+\left\lfloor\frac{k}{2}\right\rfloor$.

Proof. Let $D$ be an asymmetric primitive two-coloured cylce of length $n$. Since $D$ is primitive, then $n$ must be odd and $n \geq 3$. Lemma 2.1 and Lemma 2.2 imply that $\exp _{D}\left(v_{k}\right)=\frac{1}{4}\left(n^{2}-1\right)+\left\lfloor\frac{k}{2}\right\rfloor$. 


\section{REFERENCES}

[1] B. L. Shader and S. Suwilo, "Exponent of nonnegative matrix pairs," Linear Algebra Appl., vol. 363, pp. 275-293, 2003.

[2] S. Suwilo, "2-exponents of two-coloured lollipops," Linear Algebra Appl., vol. 21, pp. 11-22, 2008.

[3] Y. Gao and Y. Shao, "Generalized exponents of primitive two-colored digraph," Linear Algebra Appl., vol. 430, pp. 1550-1565, 2009.

[4] V. M. Fomichev and Y. E. Avezova, "The exact formula for the exponents of the mixing digraphs of register transformations," Journal of Applied and Industrial Mathematics, vol. 2, 2011.

[5] S. Suwilo, "Vertex exponent of two-colored primitive extermal ministrong digraphs," Global Journal of Technology and Optimization, vol. 14, pp. 308-320, 2020.

[6] A. Syahmarani and S. Suwilo, "Vertex exponents of a class of two-colored hamiltonian digraphs," Journal of the Indonesian Mathematical Society, vol. 18, pp. 1-19, 2012. 